Abanico Veterinario. Enero-Diciembre 2021; 11:1-11. http://dx.doi.org/10.21929/abavet2021.18

Artículo Original. Recibido: 05/09/2020. Aceptado: 25/03/2021. Publicado: 11/05/2021. Clave: e2020-77.

\title{
El empleo de licor y harina de germen de maíz en dietas para pollos de engorda
}

\author{
Employment of steep liquor and corn germ meal on broiler diets
}

\section{Rodríguez-López Nayeli11 , Ávila-González Ernesto ${ }^{11 \mathrm{D}}$, López-Coello Carlos ${ }^{11 \mathrm{D}}$, Arce-Menocal José ${ }^{\star 21 D}$, Pérez-Malave Victor ${ }^{31 D}$, Cortes-Cuevas Arturo ${ }^{11 D}$, Herrera- Camacho José ${ }^{I D}$}

1 Universidad Nacional Autónoma de México, CDMX, México. ${ }^{2}$ Universidad Michoacana de San Nicolás de Hidalgo Morelia, Posta Veterinaria. Carretera Morelia-Zinapécuaro. Colonia El Trébol, Tarímbaro, Michoacán, México. ${ }^{3}$ ADM Animal Nutrition, Quincy, Illinois, Estados Unidos de América. *Autor de correspondencia Arce-Menocal José._mvznayeli_rguez@hotmail.com, avilaernesto@yahoo.com coelloca@servidor.unam.mx, josearce_55@yahoo.com.mx, vperez@amn.com, cortescuevasarturo@yahoo.com, jose.camacho@umich.mx

\section{Resumen}

El objetivo del estudio fue evaluar en dietas de pollos, el valor alimenticio de la mezcla de harina de germen de maíz (HGM) en 50\% y la adición de 50 \% de licor de maíz (LM) sin y con enzimas carbohidrasas (CH) y la fermentación durante 24 horas. Se realizó un ensayo con el LM + enzimas para Polisacáridos no amiláceos (PNA) y LM con fermentación por 24 h. Se utilizaron 240 pollos machos Ross 708, de 6 a 50 días de edad alimentados con dietas base maíz + pasta de soya (iniciación, crecimiento y finalización), con 5,10 y $20 \%$ de HGM, con la inclusión de diferentes presentaciones de LM (0 \% LM, LM natural y LM fermentado) en porcentajes iguales a los de HGM sin y con enzimas carbohidrasas (103 $000 \mathrm{U} / \mathrm{g}$ de xilanasas, $128000 \mathrm{U} / \mathrm{g}$ de celulasas y $33000 \mathrm{U} / \mathrm{g}$ de beta-glucanasas) a $25 \mathrm{ppm}$. La ganancia de peso de los pollos de 6 a 50 días de edad mejoró $5 \%(P<0.05)$ con la adición de LM natural al HGM. La combinación HGM + LM natural, incrementó el valor alimenticio en dietas para pollo de engorda. La adición de enzimas en dietas con HGM+LM natural no afectaron el comportamiento productivo de los pollos.

Palabras clave: licor de maíz, harina de germen de maíz, enzimas, pollos de engorda.

\begin{abstract}
The objective of the study was to evaluate in broiler diets, the feeding value of corn germ meal (CGM) mixture at $50 \%$ and the addition of $50 \%$ corn liquor $(\mathrm{CL})$ without and with carbohydrase enzymes $(\mathrm{CH})$ and fermentation for 24 hours. A trial with $\mathrm{CL}+$ enzymes for non-starch polysaccharides (NSP) and CL with fermentation for $24 \mathrm{~h}$ was carried out. A total of 240 male Ross 708 broilers were used, from 6 to 50 days of age fed corn + soybean paste based diets (starter, grower and finisher), with 5, 10 and $20 \%$ CGM, with the inclusion of different presentations of $\mathrm{CL}(0 \% \mathrm{CL}$, natural $\mathrm{CL}$ and fermented $\mathrm{CL})$ in percentages equal to those of CGM without and with carbohydrase enzymes (103 $000 \mathrm{U} / \mathrm{g}$ of xylanases, $128000 \mathrm{U} / \mathrm{g}$ of cellulases and $33000 \mathrm{U} / \mathrm{g}$ of beta-glucanases) at $25 \mathrm{ppm}$. Weight gain of 6- to 50-day-old chicks improved $5 \%(P<0.05)$ with the addition of natural $C L$ to $C G M$. The combination of CGM + natural $C L$ increased feed value in broiler diets. The addition of enzymes in diets with CGM + natural CL did not affect broiler performance.
\end{abstract}

Keywords: corn liquor, corn germ meal, fermentation, enzymes, broiler chickens. 


\section{INTRODUCCIÓN}

Las dietas de pollos de engorda están compuestas tradicionalmente de maíz o sorgo como principales fuentes de energía y pasta de soya como fuente de proteína (Knudsen, 2014); sin embargo, en los últimos años el maíz se ha desviado para la producción de etanol, alcanzado precios sin precedentes (Donohue y Cunningham, 2009). La harina de germen de maíz (HGM) y el licor de maíz (LM) son co-productos originados a partir de la molienda húmeda del maíz; ambos pueden ser utilizados solo o en combinación como ingredientes alternativos en dietas para aves y cerdos (Davis, 2001; Rojas et al., 2013, Albuquerque et al., 2014). Una gran variedad de co-productos pueden ser utilizados en dietas para aves y cerdos (Rojas et al., 2013); su inclusión puede ayudar a reducir el costo de alimentación (Rochell et al., 2011). No obstante, la abundante disponibilidad de estos co-productos en la agroindustria por sus precios bajos y su inclusión en las dietas avícolas se ha visto limitada debido a la presencia de polisacáridos no amiláceos (PNA), debido a que aumentan la viscosidad intestinal, afectan el crecimiento y el rendimiento productivo de las aves (Malathi y Devegowda, 2001). Los PNA consisten en una serie de polisacáridos solubles e insolubles presentes en la pared celular (Vincken et al., 2003). Los principales polisacáridos en los granos de cereales son los arabinoxilanos (AX) y $\beta$ glucanos.

Con el objetivo de mejorar el valor nutritivo de las dietas avícolas (Kaczmarek et al., 2014), se han utilizado enzimas exógenas que actúan sobre las cadenas de los polímeros que causan su ruptura en partículas más pequeñas (Kaczmarek et al., 2014; Castro et al., 2020). Cuando ocurre un decremento del valor de energía de los ingredientes que integra las dietas de pollo, la utilización de enzimas que degradan los PNA como las xilanasas, son utilizadas (O'Neill et al., 2012).

Por lo tanto, el objetivo de este estudio fue evaluar el HGM+LM (50 y 50\%), sin y con la fermentación durante 24 horas; además sin y con la inclusión de carbohidrasas para PNA, en dietas para pollos de engorda.

\section{MATERIAL Y MÉTODOS}

El trabajo en pollos de engorda, se llevó a cabo en el Centro de Investigación de ADM en Quincy, Illinois, Estados Unidos de América. Coordenadas 39 $55^{\prime} 56^{\prime \prime}$ de LN y $91^{\circ} 23^{\prime}$ 19" de LO, altitud 193 msnm.

\section{Análisis de laboratorio}

En el laboratorio de Investigación ADM Animal Nutrition en Quincy, Illinois se realizaron los análisis de humedad, materia seca (MS), fibra detergente neutra (FND), fibra detergente ácida (FAD), proteína cruda ( $P C$ ), extracto etéreo ( $E E)$, lignina y almidón. También de licor de maíz para el análisis de cenizas, humedad, $\mathrm{pH}$, fibra detergente neutra (FND), fibra detergente ácida (FAD), PC, EE y almidón. 
Se utilizaron un total de 350 pollos machos Ross 708 de un día de edad, que fueron adquiridos de la incubadora Welp en Bancroft, lowa. Se seleccionaron 240, en base al peso corporal inicial, eligiendo pollos a los 5 días de edad entre los 94 y $114 \mathrm{~g}$. Antes de dar inicio a este estudio, todas las aves fueron identificadas con bandas individuales en el ala.

Cuadro 1. Composición de las dietas basales en tres fases de alimentación para pollos de engorda

\begin{tabular}{|c|c|c|c|}
\hline Ingrediente \% & Iniciación & Crecimiento & Finalización \\
\hline Maíz \% & 49.98 & 44.38 & 36.03 \\
\hline Pasta de soya $47.5 \%$ & 37.05 & 32.45 & 25.60 \\
\hline Aceite de soya (desgomado) & 2.50 & 4.50 & 6.00 \\
\hline HGM & 5.00 & 10.00 & 20.00 \\
\hline LM & ----- & ----- & ----- \\
\hline Agua & 2.35 & 4.95 & 9.00 \\
\hline $\mathrm{CH}, \mathrm{g}^{1}$ & ----- & ----- & ----- \\
\hline L-Lisina-HCI $98 \%$ & 0.12 & ---- & ----- \\
\hline DL- Metionina $99.5 \%$ & 0.14 & 0.08 & 0.04 \\
\hline L- Treonina $98.5 \%$ & 0.07 & ----- & ----- \\
\hline Carbonato de calcio $38 \%$ & 1.40 & 1.35 & 1.45 \\
\hline Fosfato monocálcico 21 \% & 1.65 & 1.55 & 1.15 \\
\hline Sal & 0.35 & 0.35 & 0.35 \\
\hline Bicarbonato de sodio & 0.10 & 0.10 & 0.10 \\
\hline Sulfato de cobre $25.2 \%^{2}$ & 0.00 & 0.00 & 0.00 \\
\hline Sulfato de hierro $30 \%{ }^{2}$ & 0.01 & 0.01 & 0.01 \\
\hline Selenio $0.06 \%$ & 0.05 & 0.05 & 0.05 \\
\hline Cloruro de colina $70 \%$ & 0.05 & 0.04 & 0.03 \\
\hline Coban $200^{3}$ & 0.05 & 0.05 & 0.05 \\
\hline Premezcla de Minerales* & 0.03 & 0.03 & 0.03 \\
\hline Premezcla de Vitaminas ${ }^{\star *}$ & 0.10 & 0.10 & 0.10 \\
\hline Nutriente & \multicolumn{3}{|c|}{ Análisis calculado } \\
\hline $\mathrm{EM}, \mathrm{Kcal}^{-1}$ & 2,894 & 2,885 & 2,741 \\
\hline Materia seca \% & 86.01 & 83.99 & 80.76 \\
\hline Humedad \% & 13.99 & 16.01 & 19.24 \\
\hline Proteína cruda \% & 22.27 & 20.50 & 19.00 \\
\hline Extracto etéreo \% & 4.60 & 6.57 & 7.88 \\
\hline Fibra cruda \% & 2.73 & 2.77 & 3.21 \\
\hline Fibra detergente neutra \% & 8.52 & 9.81 & 12.63 \\
\hline Calcio \% & 0.98 & 0.96 & 0.91 \\
\hline Fósforo disponible \% & 0.46 & 0.45 & 0.36 \\
\hline Lisina digestible \% & 1.23 & 1.04 & 0.92 \\
\hline Metionina digestible \% & 0.46 & 0.39 & 0.33 \\
\hline
\end{tabular}

* La premezcla proporciona por kg: Cobre $25 \mathrm{mg}$, Yodo $30 \mathrm{mg}$, Manganeso $90 \mathrm{mg}$, Selenio $0.3 \mathrm{mg}$, Zinc 100 mg, Excipiente cbp. $100.0 \mathrm{~g} ;{ }^{* *}$ La premezcla proporciona por kg: Vitamina A 12000 UI, Vitamina D3 $3500 \mathrm{UI}$, Vitamina E $16 \mathrm{UI}$, Biotina $0.2 \mathrm{mg}$, Colina $300 \mathrm{mg}$, Ácido fólico $1 \mathrm{mg}$, Vehículo cbp. $1.000 .00 \mathrm{~g}$. ${ }^{1}$ Inclusion de enzimas $\mathrm{CH}$ se realizó sin ningún valor nutricional, ${ }^{2}$ Aditivos promotores del crecimiento, ${ }^{3}$ Coccidiostato. 
Se tuvo un total de 60 corrales, con 4 pollos cada uno, de $56 \mathrm{~cm}$ de largo por $102 \mathrm{~cm}$ de ancho, con pisos de alambre; acondicionando viruta de madera como material de cama y temperatura controlada con termostato. Los pollos fueron mantenidos en tres fases de alimentación, las dietas experimentales fueron fabricadas en la planta de alimentos piloto de ADM en forma de harina para iniciación, crecimiento y finalización: de 6 a 13, de 14 a 28 y de 29 a 50 días (cuadro 1).

Las carbohidrasas utilizadas tenían una actividad enzimática de $103000 \mathrm{U} / \mathrm{g}$ de xilanasa, $128000 \mathrm{U} / \mathrm{g}$ de celulasa y $33000 \mathrm{U} / \mathrm{g}$ de $\beta$-glucanasa, con dosis de 25 ppm.

La inclusión de harina de germen de maíz y licor de maíz en las dietas fue al 5, 10 y 20 $\%$ en las tres fases de alimentación: iniciación, crecimiento y finalización, respectivamente; se mantuvieron con una relación 1:1. En los alimentos de los tratamientos 1 y 4, se les añadió agua, con la finalidad de contar con la misma dilución de energía como en aquellas dietas que contenían licor de maíz. En el tratamiento 3 se realizó la mezcla HGM + LM, adicionando las enzimas $\mathrm{CH}$ para PNA; en el tratamiento 6 las mezclas se colocaron en un recipiente de plástico hermético y se dejaron reposar por un periodo de $24 \mathrm{~h}$ a temperatura ambiente. Todas las dietas tenían un contenido similar de nutrientes.

\section{Registro de consumo de alimento}

El pesaje de alimento y de los pollos se realizó al llegar las aves a la granja, al inicio y final de cada fase de alimentación.

\section{Registro de las temperaturas ambientales en la caseta de ambiente controlado}

Las lecturas de las temperaturas mínimas y máximas a nivel de los pollos con el empleo de termómetros digitales se muestran en el cuadro 2. A las aves se les proporcionó un calendario de luz artificial, el cual consistió en dar 23 horas luz/día la primera semana, 14 la segunda, 12 la tercera, 10 la cuarta y 8 de la quinta hasta el final de la prueba.

Cuadro 2. Temperaturas mínimas y máximas registradas en el experimento in vivo con pollos

\begin{tabular}{ccc}
\hline Edad (días) & Mínimas $\left({ }^{\circ} \mathbf{C}\right)$ & Máximas $\left({ }^{\circ} \mathbf{C}\right)$ \\
\hline $1-8$ & 29 & 32 \\
$9-15$ & 27 & 29 \\
$16-24$ & 24 & 26 \\
$25-32$ & 22 & 24 \\
$33-50$ & 19 & 21 \\
\hline
\end{tabular}




\section{Parámetros zootécnicos evaluados}

Se llevaron durante el transcurso del estudio, registros de la ganancia de peso corporal (g), consumo de alimento (g) y conversión alimenticia $(\mathrm{g} / \mathrm{g})$; la cual se obtuvo dividiendo la cantidad de alimento consumido entre la ganancia de peso obtenido en cada fase de alimentación.

\section{Eliminación de animales}

Se excluyeron del experimento aves enfermas o muertas; posteriormente fueron pesadas individualmente todas las aves de dicho corral y el comedero para realizar el cálculo de ajuste de las variables productivas. Para el consumo de alimento corregido a mortalidad, se realizó a través de la resta del consumo de alimento de las aves muertas con el consumo de alimento total.

\section{Análisis estadístico}

Los resultados obtenidos para ganancia de peso, consumo de alimento y conversión alimenticia en cada fase de alimentación, se analizaron con un diseño experimental completamente al azar con arreglo factorial $2 \times 3$, factor $A$ (con y sin enzimas) y factor $B$ (LM 0\%, LM natural y LM fermentado); la comparación de medias se realizó usando la prueba de Tukey ( $\mathrm{P} \leq 0.05)$. Para analizar los datos se utilizó el paquete estadístico SAS (SAS, 2012).

\section{RESULTADOS}

El perfil nutricional de los ingredientes utilizados en el ensayo mostró para el maíz $8.3 \%$ de PC, un $94.9 \%$ de MS, $5.1 \%$ de humedad, $82.7 \%$ de almidón y contó con una baja cantidad de fibra (2.9\% de FDN y $8.0 \%$ de FAD). La HGM con $25.7 \%$ de PC, $92.0 \%$ de MS, un porcentaje considerable de almidón (26.2 \%), de aceite (1.7\%); además, de ser un ingrediente rico en fibra debido que posee un $13.0 \%$ de FDN y $38.3 \%$ de FAD. Por otro lado, el LM es un ingrediente líquido con $48.9 \%$ de humedad, un $\mathrm{pH}$ de 3.58 proporcionado por la cantidad de ácido láctico con el que ésta cuenta (11.6\%); asimismo, posee una cantidad alta de PC (30.0\%) y de aceite (4.5\%). La combinación de 50\% HGM y $50 \%$ LM (uno a uno), resulta atractiva en los análisis químicos realizados por su concentración promedio en cuanto de PC $(27.9 \%)$ y almidón (18.9 \%).

En el cuadro 3, se presentan los resultados obtenidos para ganancia de peso de 6 a 50 días de edad. No se observa diferencia estadística $(p>0.05)$ para el factor enzimas en las distintas fases de alimentación; sin embargo, para el factor dietas se aprecia que la mezcla de LM+HGM incrementó significativamente $(\mathrm{P}<0.05)$ la ganancia de peso a partir de los 29 días de edad, siendo mejor este efecto cuando no fue fermentada la combinación. No se encontraron efectos de interacción $(p>0.05)$ de enzimas $x$ dietas en las fases de alimentación. 
El cuadro 4, muestra los datos del consumo de alimento de 6 a 50 días de edad. Para el factor enzimas no existió diferencia estadística $(P>0.05)$; sin embargo, para el factor dietas se aprecia que las mezclas de LM+HGM incrementaron el consumo de alimento significativamente $(\mathrm{P}<0.05)$ a partir de los 29 días de edad. No se encontraron efectos de interacción $(P>0.05)$ de enzimas $x$ dietas en las fases de alimentación.

En el cuadro 5, se presentan los valores obtenidos para conversión alimenticia de 6 a 50 días de edad. No se observa diferencia estadística $(P>0.05)$ para los factores enzimas y dietas ni efectos de interacción entre los mismos. La diferencia observada $(P<0.05)$, fue en la fase de crecimiento a favor de la dieta control.

Cuadro 3. Resultados en pollos de engorda de 6 a 50 días de edad, alimentados con la mezcla de LM+HGM con y sin enzimas

\begin{tabular}{|c|c|c|c|c|}
\hline \multicolumn{5}{|c|}{ Ganancia de peso $(\mathrm{g})$} \\
\hline Enzimas $\mathrm{CH}$ & Control & LM+HGM & $\begin{array}{c}\text { LM+HGM } \\
\text { Fermentado }\end{array}$ & Promedio \\
\hline \multicolumn{5}{|c|}{ Fase de Iniciación } \\
\hline $\operatorname{Sin}$ & 193 & 192 & 195 & $193 \pm 2^{\mathrm{a}}$ \\
\hline Con & 189 & 192 & 194 & $191 \pm 2^{a}$ \\
\hline Promedio & $191 \pm 3^{a}$ & $192 \pm 3^{a}$ & $194 \pm 3^{a}$ & \\
\hline \multicolumn{5}{|c|}{ Fase de Crecimiento } \\
\hline $\operatorname{Sin}$ & 882 & 881 & 869 & $877 \pm 6^{a}$ \\
\hline Con & 867 & 864 & 872 & $868 \pm 6^{a}$ \\
\hline Promedio & $874 \pm 7^{a}$ & $872 \pm 8^{a}$ & $871 \pm 7^{a}$ & \\
\hline \multicolumn{5}{|c|}{ Fase de finalización } \\
\hline $\operatorname{Sin}$ & 1702 & 1845 & 1723 & $1756 \pm 21^{a}$ \\
\hline Con & 1688 & 1839 & 1786 & $1771 \pm 22^{a}$ \\
\hline Promedio & $1695 \pm 26^{a}$ & $1842 \pm 26^{b}$ & $1754 \pm 27^{a}$ & \\
\hline \multicolumn{5}{|l|}{ Acumulado } \\
\hline $\operatorname{Sin}$ & 2776 & 2917 & 2787 & $2827 \pm 35^{a}$ \\
\hline Con & 2744 & 2904 & 2856 & $2835 \pm 35^{a}$ \\
\hline Promedio & $2760 \pm 30^{a}$ & $2911 \pm 30^{b}$ & $2821 \pm 31^{a b}$ & \\
\hline
\end{tabular}

\section{DISCUSIÓN}

La HGM es un ingrediente fibroso con una baja digestibilidad in vitro de la materia seca $(61.25 \%$ ), que posee una energía metabolizable de $1650 \mathrm{Kcal} / \mathrm{Kg}$ (Archer Daniels Midland Company, 2016). Debido a la presencia del germen en la HGM, se determinó en los análisis de laboratorio en esta muestra un porcentaje de $26.23 \%$ de almidón, valor superior al obtenido por Rochell et al. (2011) de $15.29 \%$; además posee un $1.65 \%$ de aceite, caracterizándolo como un ingrediente energético, como lo describieron Milošević et al. (2011). En otro estudio realizado por Rochell et al. (2011) determinó que la HGM 
cuenta con $10.87 \%$ de humedad, valor similar al reportado en este análisis (7.93 \%). Por otro lado, Rojas et al. (2013) realizaron un estudio donde determinaron el valor de EB de la HGM, el cual fue de $4184 \mathrm{Kcal}^{-1}$, valor inferior al obtenido por Rochell et al. (2011) de $4767 \mathrm{Kcal}^{-1}$, teniendo la muestra de este estudio un valor intermedio a dichos resultados que fue de $4573 \mathrm{Kcal}^{-1}$.

Cuadro 4. Resultados en pollos de engorda de 6 a 50 días de edad, alimentados con la mezcla de LM+HGM con y sin enzimas

\begin{tabular}{ccccc}
\hline \multicolumn{4}{c}{ Consumo de alimento (g) } \\
\hline Enzimas CH & Control & LM+HGM & $\begin{array}{c}\text { LM+HGM } \\
\text { Fermentado }\end{array}$ & Promedio \\
\hline Fase de Iniciación & & & \\
\hline Sin & 260 & 260 & 261 & $261 \pm 3^{\mathrm{a}}$ \\
Con & 249 & 257 & 257 & $254 \pm 3^{\mathrm{a}}$ \\
Promedio & $255 \pm 4^{\mathrm{a}}$ & $259 \pm 4^{\mathrm{a}}$ & $259 \pm 4^{\mathrm{a}}$ & \\
\hline Fase de Crecimiento & & & $1273 \pm 9^{\mathrm{a}}$ \\
\hline Sin & 1258 & 1291 & $1258 \pm 9^{\mathrm{a}}$ \\
Con & 1250 & 1260 & 1264 & \\
Promedio & $1254 \pm 11^{\mathrm{a}}$ & $1275 \pm 11^{\mathrm{a}}$ & $1267 \pm 11^{\mathrm{a}}$ & \\
\hline Fase de finalización & & & $3717 \pm 38^{\mathrm{a}}$ \\
\hline Sin & 3643 & 3842 & 3667 & $3734 \pm 39^{\mathrm{a}}$ \\
Con & 3546 & 3858 & 3797 & \\
Promedio & $3595 \pm 47^{\mathrm{a}}$ & $3850 \pm 47^{\mathrm{b}}$ & $3732 \pm 48^{\mathrm{b}}$ & \\
\hline Acumulado & & & $5251 \pm 62^{\mathrm{a}}$ \\
\hline Sin & 5161 & 5394 & 5198 & $5260 \pm 62^{\mathrm{a}}$ \\
Con & 5045 & 5392 & 5342 & \\
Promedio & $5103 \pm 53^{\mathrm{a}}$ & $5393 \pm 53^{\mathrm{b}}$ & $5270 \pm 55^{\mathrm{b}}$ & \\
\hline
\end{tabular}

$\overline{a, b}$ en la misma fila indica valores diferentes $(\mathrm{P}<0.05)$

Por otro lado, el licor de maíz es un ingrediente altamente digestible como lo demuestran los resultados de la muestra analizada (99.19\%) y los reportes que indican un contenido de energía metabolizable de $1595 \mathrm{Kcal}^{-1}$ (Tekchandani et al., 1999, Sultan et al., 2017). El uso de $\mathrm{CH}$ para PNA en dietas para pollos de engorda no incrementó el consumo de alimento (Zhang et al., 2014), de igual manera se observó en el presente experimento con la inclusión de $\mathrm{CH}$, de los 6 a los 50 d de edad.

Por otro lado, la utilización de $50 \%$ de LM+50\% de HGM estimuló el consumo de alimento de los 6 a los $50 \mathrm{~d}$ de edad, probablemente por un incremento de la palatabilidad de las dietas. En estudios realizados en otras especies (cerdos y ganado) que utilizaron LM se ha observado un mayor consumo de alimento relacionado al incremento de la 
palatabilidad de la dieta (Lusby et al., 1981), así como otros sustratos ricos en carbohidratos (Medina et al., 2014).

Ha sido ampliamente estudiado la utilización de $\mathrm{CH}$ para PNA en pollos de engorda y su efecto en el aumento de la ganancia de peso (Adeola y Cowieson, 2011); no obstante, no se presentó un incremento en esta variable de los 6 a los $50 \mathrm{~d}$ de edad; probablemente debido a que se subestimó la dosificación de la $\mathrm{CH}$ para PNA utilizada en este estudio. Además, no se encontraron diferencias en la ganancia de peso, de los 6 a los $50 \mathrm{~d}$ de edad, cuando se utilizó la inclusión de LM fermentado a 24 h. El LM utilizado en este estudio tuvo un $\mathrm{pH}$ de 3.8, probablemente la falta de efecto en la ganancia de peso en el tratamiento LM fermentado a $24 \mathrm{~h}$, fue a lo descrito anteriormente.

Cuadro 5. Resultados en pollos de engorda de 6 a 50 días de edad, alimentados con la mezcla de LM+HGM con y sin enzimas

\begin{tabular}{|c|c|c|c|c|}
\hline \multicolumn{5}{|c|}{ Conversión alimenticia $(\mathbf{g} / \mathbf{g})$} \\
\hline Enzimas $\mathrm{CH}$ & Control & LM+HGM & $\begin{array}{c}\text { LM+HGM } \\
\text { Fermentado }\end{array}$ & Promedio \\
\hline \multicolumn{5}{|c|}{ Fase de Iniciación } \\
\hline $\operatorname{Sin}$ & 1.35 & 1.36 & 1.34 & $1.35 \pm 0.11^{\mathrm{a}}$ \\
\hline Con & 1.31 & 1.34 & 1.33 & $1.33 \pm 0.11^{\mathrm{a}}$ \\
\hline Promedio & $1.33 \pm 0.01^{\mathrm{a}}$ & $1.35 \pm 0.01^{\mathrm{a}}$ & $1.33 \pm 0.01^{\mathrm{a}}$ & \\
\hline \multicolumn{5}{|c|}{ Fase de Crecimiento } \\
\hline $\operatorname{Sin}$ & 1.43 & 1.47 & 1.46 & $1.44 \pm 0.006^{\mathrm{a}}$ \\
\hline Con & 1.44 & 1.46 & 1.45 & $1.44 \pm 0.006^{\mathrm{a}}$ \\
\hline Promedio & $1.43 \pm 0.007^{a}$ & $1.46 \pm 0.007^{b}$ & $1.46 \pm 0.007^{b}$ & \\
\hline \multicolumn{5}{|c|}{ Fase de finalización } \\
\hline $\operatorname{Sin}$ & 2.15 & 2.09 & 2.13 & $2.12 \pm 0.016^{a}$ \\
\hline Con & 2.10 & 2.10 & 2.13 & $2.11 \pm 0.016^{\mathrm{a}}$ \\
\hline Promedio & $2.13 \pm 0.019^{a}$ & $2.10 \pm 0.019^{a}$ & $2.13 \pm 0.020^{a}$ & \\
\hline \multicolumn{5}{|l|}{ Acumulado } \\
\hline $\operatorname{Sin}$ & 1.86 & 1.85 & 1.87 & $1.86 \pm 0.01^{a}$ \\
\hline Con & 1.84 & 1.86 & 1.87 & $1.86 \pm 0.01^{a}$ \\
\hline Promedio & $1.85 \pm 0.01^{a}$ & $1.85 \pm 0.01^{\mathrm{a}}$ & $1.87 \pm 0.01^{\mathrm{a}}$ & \\
\hline
\end{tabular}

$\overline{a, b}$ en la misma fila indica valores diferentes $(\mathrm{P}<0.05)$

Una característica de la HGM es la capacidad de absorber nutrientes líquidos (Davis, 2001, Giannenas et al., 2017). Cabe mencionar que el LM evaluado en este estudio tenía un porcentaje alto de PC (29.97\%), extracto etéreo (4.49\%) y almidón (11.53\%). Probablemente el empleo de LM al mezclarlo con HGM incrementó la biodisponibilidad de nutrientes, con lo que se mejoró la ganancia de peso de los pollos en (2911 vs $2760 \mathrm{~g}$ ), lo que corresponde a un $5 \%$; sugiriendo que esta combinación tiene un alto potencial para su empleo en dietas para pollos. 


\section{CONCLUSIONES}

La combinación de 50\% de HGM + 50\% de LM natural, en dietas de iniciación (5\%), crecimiento (10\%) y finalización (20\%), incrementó la ganancia de peso y el consumo de alimento en pollos de engorda, debido a su digestibilidad y palatabilidad. La adición de enzimas o la fermentación con HGM+LM no mejoraron el comportamiento productivo de los pollos.

\section{Agradecimientos}

Los autores expresan su sincero agradecimiento y aprecio a la Universidad Nacional Autónoma de México por el apoyo académico, así como a James Dunn por su contribución y a ADM Animal Nutrition por haber proporcionado sus instalaciones para la realización de estos estudios y por el financiamiento del presente proyecto.

\section{LITERATURA CITADA}

ADEOLA O, Cowieson AJ. 2011. Board-invited review: Opportunities and challenges in using exogenous enzymes to improve nonruminant animal production. Journal Animal Science. 89 (10):3189-3218. https://doi.org/10.2527/jas.2010-3715

ALBUQUERQUE CS, Rabello CBV, Santos MJB, Lima MB, Silva EP, Lima TS, Ventura DP, Dutra Jr WM. 2014. Chemical composition and metabolizable energy values of corn germ meal obtained by wet milling for layers. Brazilian Journal of Poultry Science. 16 (1): 107-112. https://doi.org/10.1590/S1516-635X2014000100015

ARCHER DANIELS MIDLAND COMPANY. 2016. Feed \& Pet Food. Ingredients Catalog. Decatur, IL, USA.

http://www.admanimalnutrition.com/webcenter/portal/ADMAnimalNutrition/pages_anhom e

CASTRO FS, Bertechini AG, Machado CLE, Sampaio CAH, Gonçalves FVG, Carrera CJC. 2020. Effect of different levels of supplementary alpha-amylase in finishing broilers. Acta Scientiarum Animal Sciences. 42: e47546. 2019. https://doi.org/10.4025/actascianimsci.v42i1.47546

DAVIS KS. 2001. Corn milling, processing and generation of co-products. Minnesota Corn Grower Association. Technical Symposium. September 11, 2001. https://www.biofuelscoproducts.umn.edu/sites/biodieselfeeds.cfans.umn.edu/files/ddgstechinfo-pro-20.pdf 
DONOHUE M, Cunningham DL. 2009. Effects of grain and oilseed prices on the costs of US poultry production. Journal Applied Poultry Research. 18 (2): 325-337. https://doi.org/10.3382/japr.2008-00134

GIANNENAS I, Bonos E, Anestis V, Filioussis G, Papanastasiou DK, Bartzanas T, Papaioannou N, Tzora A, Skoufos I. 2017 Effects of protease addition and replacement of soybean meal by corn gluten meal on the growth of broilers and on the environmental performances of a broiler production system in Greece. PLOS ONE. 12(1): 1-26. https://doi.org/10.1371/journal.pone.0169511

KACZMAREK SA, Rogiewicz A, Mogielnicka M, Rutkowski A, Jones RO, Slominski BA. 2014. The effect of protease, amylase, and nonstarch polysaccharide-degrading enzyme supplementation on nutrient utilization and growth performance of broiler chickens fed corn-soybean meal-based diets. Poultry Science. 93 (7): 1745-1753. https://doi.org/10.3382/ps.2013-03739

KNUDSEN K. 2014. Fiber and nonstarch polysaccharide content and variation in common crops used in broiler diets. Poultry Science. 93 (7): 2380-2393. https://doi.org/10.3382/ps.2014-03902

LUSBY KS, Armbruster SL, Dvorak MJ. 1981. Condensed molasses soluble and corn steep liquor as protein supplements for range cows. Animal Science Research Report. 57:40-46. https://docplayer.net/50774428-Condensed-molasses-solubles-and-cornsteep-liquor-as-protein-supplements-for-range-cows.html

MALATHI V, Devegowda G. 2001. In vitro evaluation of nonstarch polysaccharide digestibility of feed ingredients by enzymes. Poultry Science. 80 (3): 302-305. https://doi.org/10.1093/ps/80.3.302

MEDINA MN, González CA, Daza LS, Restrepo O, Barahona R. 2014. Desempeño productivo de pollos de engorde suplementados con biomasa de Saccharomyces cerevisiae derivada de la fermentación de residuos de banano. Revista de la Facultad de Medicina Veterinaria y de Zootecnia. 61(3): 270-283. http://dx.doi.org/10.15446/rfmvz.v61n3.46873

MILOŠEVIĆ N, Stanačev V, Nikolova N, Pavlovski Z. 2011. Corn meal in broiler chicken nutrition. Macedonian Journal Animal Science. 1 (1); 107-111. https://doi.org/10.2298/bah0606071m 
O'NEILL HV, Mathis G, Lumpkins BS, Bedford MR. 2012. The effect of reduced calorie diets, with and without fat, and the use of xylanase on performance characteristics of broilers between 0 and 42 days. Poultry Science. 91 (6): 1356-1360. https://doi.org/10.3382/ps.2011-01867

ROCHELL SJ, Kerr BJ, Dozier WA III. 2011. Energy determination of corn co-products fed to broiler chicks from 15 to 24 days of age, and use of composition analysis to predict nitrogen-corrected apparent metabolizable energy. Poultry Science. 90 (9): 1999-2007. https://doi.org/10.3382/ps.2011-01468

ROJAS OJ, Liu Y, Stein HH. 2013. Phosphorus digestibility and concentration of digestible and metabolizable energy in corn, corn coproducts, and bakery meal fed to growing pigs. Journal Animal Science. 91 (11): 5326-5335. https://doi.org/10.2527/jas.2013-6324

SAS Institute. 2012. Statistical Analysis Software SAS/STATR. Version 9.0.2, Cary, N.C. USA. SAS Institute Inc. ISBN 978-1-60764-599-3. http://www.sas.com/en_us/software/analytics/stat.html\#

SULTAN JI, lqbal Z, Kamran Z, Shahid A, Ali R, Ahmad S, Ali A, Koutoulis KC, Shahzad MI, Ahsan U, Shahid I. 2017. Effect of corn replacement with enzose (corndextrose) on growth performance and nutrient digestibility in broilers. Journal of Applied Poultry Research. 26:383-390. http://dx.doi.org/10.3382/japr/pfx006

TEKCHANDANI HK, Dias FF, Mehta D. 1999. Maize wet milling co-products as feed additives: Perspective and opportunities. Journal Scientific Industrial Research. 58: 8388. Corpus ID: 56047269. http://nopr.niscair.res.in/handle/123456789/17796

VINCKEN JP, Schols HA, Oomen RJ, McCann MC, Ulvskov P, Voragen AG, Visser, RG. 2003. If homogalacturonan were a side chain of rhamnogalacturonan I. Implications for cell wall architecture. Plant Physiology. 132 (4): 1781-789. https://doi.org/10.1104/pp.103.022350

ZHANG L, Xu J, Lei L, Jiang Y, Gao F, Zhou GH. 2014. Effects of xylanase supplementation on growth performance, nutrient digestibility and non-starch polysaccharide degradation in different sections of the gastrointestinal tract of broilers fed wheat-based diets. Asian-Australasian Journal Animal Sciences. 27 (6): 855-861. https://doi.org/10.5713/ajas.2014.14006 